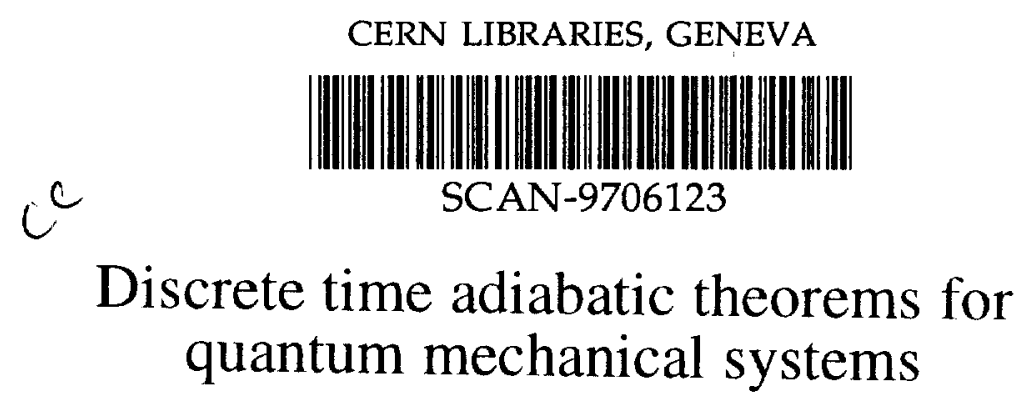

A. Dranov, J. Kellendonk, R. Seiler

Stb 288 Preprint No. 259

Diese Arbeit ist mit Unterstützung des von der Deutschen Forschungsgemeinschaft getragenen Sonderforschungsbereiches 288 entstanden und als Manuskript vervielfältigt worden.

Berlin, Mai 1997

The list of preprints of the Sonderforschungsbereich 288 is available at: http://www-sfb288.math.tu-berlin.de 


\title{
Discrete time adiabatic theorems for quantum mechanical systems
}

\author{
A. Dranov J. Kellendonk R. Seiler \\ Fachbereich Mathematik, Technische Universität Berlin, \\ 10623 Berlin, Germany
}

\begin{abstract}
The theory of adiabatic asymptotics is adapted to systems with discrete time evolution. The corresponding theorems about the approximation of physical time evolution by the adiabatic time evolution are shown to hold true in a discrete setting.
\end{abstract}

\section{Introduction}

In recent years it became more and more evident, that many concepts and results of continuous analysis have interesting counterparts in a discrete setting typically on graphs or lattices. ${ }^{1}$ An example of this kind is the one described here, the discrete version of the adiabatic theorem of quantum mechanics.

Originally formulated in the Twenties by Born and Fock [4] the adiabatic theorem has evolved through a sequence of steps. Kato $[5,6]$ has used the theorem in an important way for his analysis of perturbation theory of linear operators. Berry [2] and later Simon [8] noticed an interesting aspect of adiabatic dynamics: Adiabatic dynamics is typically geometric in the sense that it can be interpreted as parallel transport with respect to a natural connection. This point of view was important in an extension of this theorem which was motivated by the analysis of the Quantum Hall effect [1]. That article and a follow-up [7] are used in the present article as a guideline for the analysis of the discrete version of the adiabatic theorem.

In the continuous time-setting, adiabatic asymptotics applies to Hamiltonians which are time dependent in a particular way. It applies to those Hamiltonians for which there exists a large $T$ such that the variation of the Hamiltonian in time depends essentially only on the rescaled time $s=\frac{t}{T}$. The time scale set up by $T$ is supposed to be very large compared with the inverse frequencies corresponding

\footnotetext{
${ }^{1} \mathrm{~A}$ paradigm in this category is the discrete version of the theory of surfaces [3]
} 
to energy levels of the Hamiltonian at a fixed time $t$. It then makes sense to consider the limit $T \rightarrow \infty$, which is called the adiabatic limit [4]. For that, we have to consider a whole family $\{H(t, T)\}_{T}$ of time dependent Hamiltonians which is parametrized by $T$. The time dependent Schrödinger equation

$$
i \partial_{t} U(t, T)=H(t, T) U(t, T), \quad U(0, T)=1,
$$

is then more conveniently written in rescaled time $s=\frac{t}{T}$,

$$
i \partial_{s} U_{T}(s)=T H_{T}(s) U_{T}(s), \quad U_{T}(0)=1,
$$

where $U_{T}(s)=U(t, T)$ and $H_{T}(s)=H(t, T)$. In particular, $\frac{d}{d s} H_{T}(s)=T \frac{d}{d t} H(t, T)$. To repeat, the natural set up for adiabatic asymptotics is one in which $H_{T}(s)$ is independent of $T$.

The adiabatic theorems, which were considered in [1,7], apply to a situation in which the spectrum of $H(t, T)$ consists of two parts which are well separated by a gap. In that case one considers the adiabatic time evolution, which is the best approximation of the time evolution under the constraint that the spectral subspaces corresponding to the two parts are conserved. Its corresponding group of unitaries $U_{T}^{A}(s)$ is defined by the initial value problem

$$
i \partial_{s} U_{T}^{A}(s)=T H_{T}^{A}(s) U_{T}^{A}(s), \quad U_{T}^{A}(0)=1,
$$

where

$$
H_{T}^{A}(s)=H_{T}(s)+\frac{i}{T}\left[\partial_{s} P_{T}(s), P_{T}(s)\right]
$$

and $P_{T}(s)$ is the spectral projection of $H_{T}(s)$ onto one part of the spectrum. Next to the result that $U_{T}^{A^{*}} U_{T}$ is of order $T^{-1}$ the authors in $[1,7]$ show that, under the additional requirement that $H_{T}(s)$ is infinitely often differentiable, the tunneling-probability between the two parts of the spectrum is of order $T^{-\infty}$.

Our aim here is to develop the corresponding adiabatic theory for systems with discrete time evolution. For that we consider the discrete form of the Schrödinger equation, $n \in \mathbf{Z}$,

$$
U(n+1, T)=W(n, T) U(n, T), \quad U(0, T)=1,
$$

where $W(n, T)$ is a sequence of unitaries parametrized by $T$, and so is $U(n, T)$. The rescaled equation then reads, with $N=\frac{n}{T}$ and $W_{T}(N)=W(n, T)$,

$$
U_{T}\left(N+\frac{1}{T}\right)=W_{T}(N) U_{T}(N), \quad U_{T}(0)=1 .
$$

One way to obtain a discrete system is by discretizing the solution of a continuous system. In fact, upon using the time ordered product : :,

$$
W(n, T)=: e^{-i \int_{n}^{n+1} H(t, T) d t}:
$$


gives by (4) the continuous solution at discrete times. This correspondence is useful to motivate our set up. ${ }^{2}$

Like in the continuous problem, adiabatic asymptotics makes sense if one considers the (adiabatic) limit $T \rightarrow \infty$ for a family of unitaries $W_{T}(N)$ which depend weakly enough on $T$. This will mean, as we will elaborate and specify, that $W_{T}\left(N+\frac{1}{T}\right)-W_{T}(N)$ is of order $T^{-1}$. It should be kept in mind that, in the adiabatic limit, the rescaled time $N=\frac{n}{T}$ remains finite.

To consider an adiabatic evolution in the discrete setting we assume that part of the spectrum of $W_{T}(N)$ is well separated from the rest by two gaps (in a sense specified below). Let $P_{T}(N)$ then denote the spectral projection onto that part. The group of unitaries $U_{T}^{A}(N)$ describing the adiabatic evolution associated with this spectral projection ought to be defined by

$$
U_{T}^{A}\left(N+\frac{1}{T}\right)=W_{T}^{A}(N) U_{T}^{A}(N), \quad U_{T}^{A}(0)=1,
$$

for a family of unitaries $W_{T}^{A}(N)$, which shall now be determined. As in the continuous setting $U_{T}^{A}$ should preserve the eigenspace of $P_{T}(N)$, i.e.

$$
U_{T}^{A}(N) P_{T}(0)=P_{T}(N) U_{T}^{A}(N) .
$$

Furthermore, it should be close to $U_{T}$ in the sense that

$$
\begin{aligned}
P_{T}(N) U_{T}(N) U_{T}^{*}(M) P_{T}(M) & =v_{T}(N, M) U_{T}^{A}(N) U_{T}^{A^{*}}(M) P_{T}(M) \\
Q_{T}(N) U_{T}(N) U_{T}^{*}(M) Q_{T}(M) & =v_{T}(N, M) U_{T}^{A}(N) U_{T}^{A^{*}}(M) Q_{T}(M)
\end{aligned}
$$

for a positive operator $v_{T}(N, M)$. This operator is chosen to be $v_{T}(N, M)=$ $\sqrt{1-\left(P_{T}(N)-P_{T}(M)\right)^{2}}$. Using the equation of motion and the above ansatz one obtains

$$
\begin{aligned}
P_{T}\left(N+\frac{1}{T}\right) W_{T}(N) P_{T}(N) & =v_{T}\left(N+\frac{1}{T}, N\right) W_{T}^{A}(N) P_{T}(N), \\
Q_{T}\left(N+\frac{1}{T}\right) W_{T}(N) Q_{T}(N) & =v_{T}\left(N+\frac{1}{T}, N\right) W_{T}^{A}(N) Q_{T}(N),
\end{aligned}
$$

and thus

$$
W_{T}^{A}(N)=V_{T}\left(N+\frac{1}{T}, N\right) W(N)
$$

where

$$
V_{T}(N, M)=\frac{P_{T}(N) P_{T}(M)+Q_{T}(N) Q_{T}(M)}{v_{T}(N, M)} .
$$

This unitary operator has been introduced by Kato [6]. In particular, also $W_{T}^{A}(N)$ is unitary. $V_{T}$ intertwines the spectral subspaces:

$$
P_{T}(N) V_{T}(N, M)=V_{T}(N, M) P_{T}(M) .
$$

\footnotetext{
${ }^{2}$ Alternatively, we could have started with the discrete form of equation (2) which is (5). The identity $W_{T}(N)=W(n, T)$ mirrors the identity $\int_{N}^{N+1 / T} T H_{T}(s) d s=\int_{N T}^{N T+1} H(t, T) d t$ in the continuous version.
} 
A direct consequence of this equation is $P_{T}\left(N+\frac{1}{T}\right) W_{T}^{A}(N)=W_{T}^{A}(N) P_{T}(N)$ which in turn shows that the choice of $v_{T}$ is consistent with (8).

The main result of this article is the extension of the adiabatic theorems to the discrete setting. In particular, we shall first prove that the wave operator,

$$
\Omega_{T}(N):=U_{T}^{A^{*}}(N) U_{T}(N)
$$

behaves in the adiabatic limit as $\Omega_{T}(N)=1+O\left(T^{-1}\right)$. The notation $X=O\left(T^{-k}\right)$ means, as usual, that there is a constant $c$ such that $\|X\| \leq c T^{-k}$, one says that $X$ is of order $T^{-k}$.

Secondly, defining for a sequence of operators $X(N), N \in \frac{\mathrm{Z}}{T}$, the difference operator $D$ as usual by

$$
D X(N):=X\left(N+\frac{1}{T}\right)-X(N)
$$

and $D^{(2)}=D \circ D$ etc., we shall proof that if, for any $k, D^{(k)} W_{T}(N)$ is of order $T^{-k}$ then the tunneling-probability out of the spectral subspace is smaller than any inverse power of the time scale,

$$
Q_{T}(0) \Omega_{T}(N) P_{T}(0)=O\left(T^{-\infty}\right)
$$

\section{Adiabatic Theorems}

\subsection{The set up}

We consider a sequence of unitary operators $W_{T}(N), N \in \frac{\mathrm{z}}{T}$, on some Hilbertspace $\mathcal{H}$ which is parametrized by a large time $T . N=\frac{n}{T}$ plays the role of a rescaled discrete time. This sequence is supposed to satisfy the following conditions.

1 For a positive integer $k$, the value of which will be specified below, the $k$ difference of $W_{T}, D^{(k)} W_{T}(N)$, is of order $T^{-k}$.

2 The spectrum of $W_{T}(N)$ consists of two parts which are separated by two gaps in the sense that there are two continuous functions $g_{i}: \mathbf{R} \rightarrow \mathbf{S}^{\mathbf{1}}$ and an $\epsilon>0$ such that, for all $N$, $\operatorname{dist}\left(\left\{g_{1}(N), g_{2}(N)\right\}, \sigma\left(W_{T}(N)\right)\right)>\epsilon$.

Let us compare the above set up with the set up used in [1]. In that work, family of self-adjoint operators $H(s), s \in I \subset \mathbf{R}$, are investigated, which are bounded from below, $k$ times continuously differentiable and have gaps in the spectrum in the following sense: there are two continuous functions $g_{i}: \mathbf{R} \rightarrow \mathbf{R}$ and an $\epsilon>0$ such that, for all $s, \operatorname{dist}\left(\left\{g_{1}(s), g_{2}(s)\right\}, \sigma(H(s))\right)>\epsilon$. These operators $H(s)$ are in fact nothing else that $H_{T}(s)$ from (3), but they are, according to the natural adiabatic set up, independent on $T$. 
The regularity conditions which ensure the existence of a unique dynamical evolution are already built in by requiring that $W_{T}(N)$ are unitary operators. The natural adiabatic set up, in which $H_{T}$ is independent of $T$, does however not lead to a sequence $W_{T}$ of $T$-independent unitaries. In fact, assuming for simplicity that $H(t, T)$ commutes at different times, one obtains, formally, from (6)

$$
W_{T}(N)=\exp \left(-i H_{T}(N)+O\left(T^{-1}\right)\right),
$$

the $O\left(T^{-1}\right)$-term vanishing only under special circumstances. However, if $H(t, T)$ is independent of $T$ (and differentiable) then (14) shows that $D W_{T}(N)=O\left(T^{-1}\right)$. This is covered by our first condition with $k=1$. The first condition of our set up is also supported by the observation that boundedness of the $k$ th derivative of $H_{T}(s)$ (w.r.t. $s$ ) requires the $k$ th derivative of $H(t, T)$ (w.r.t. $t$ ) to be of order $T^{-k}$, and hence, to require that the $k$-difference of $W_{T}(N)$ be of order $T^{-k}$ is a natural analog in the discrete case.

It is clear that, due to the topology of $S^{1}$, one needs two functions to separate a part of the spectrum. This is not really more restrictive than the gap condition in the continuous case, however, it should be mentioned that our second condition is a priori stronger than that gap condition in case the continuous Hamiltonian $H_{T}(s)$ were unbounded, because then the spectrum of $W_{T}(N)$ could be all of $S^{1}$.

\subsection{Direct consequences of the set up}

If $\Gamma_{T}(N)$ is a contour (traversed clockwise) around the part of spectrum of $W_{T}(N)$ which lies between $g_{1}(N)$ and $g_{2}(N)$ - we assume here that we have fixed an order for these two functions, in particular that they do not level-cross - then the spectral projection $P_{T}(N)$ onto that part is given by the integral (which is to be understood in the strong sense)

$$
P_{T}(N)=\frac{1}{2 \pi i} \int_{\Gamma_{T}(N)} R_{T}(N, z) d z,
$$

where $R_{T}(N, z)=\left(W_{T}(N)-z\right)^{-1}$ is the resolvent of $W_{T}(N)$. The large $T$ behaviour of $D^{(k)} P_{T}$ is of the same order in $T$ than that of $D^{(k)} W_{T}$, since for large $T$

$$
D P_{T}(N)=-\frac{1}{2 \pi i} \int_{\Gamma_{T}} R_{T}\left(N+\frac{1}{T}, z\right) D W_{T}(N) R_{T}(N, z) d z .
$$

Here, due to condition 2 and in particular the continuity of $g_{i}$ we can choose for large enough $T$ either $\Gamma_{T}=\Gamma_{T}(N)$ or $\Gamma_{T}=\Gamma_{T}\left(N+\frac{1}{T}\right)$. This proves the case $k=1$, the one for larger $k$ following by a similar but more complicated argument.

Likewise is the large $T$ behaviour of the $k$-differences of $V_{T}(N)=V_{T}\left(N+\frac{1}{T}, N\right)$ and $W_{T}^{A}(N)$ of the same order in $T$ as that of $D^{(k)} W_{T}$, as they are defined by infinitely differentiable functions in $P$ and one may use the discrete analog of 
Leibniz rule, $D(X Y)(N)=D X(N) Y\left(N+\frac{1}{T}\right)+X(N) D Y(N)$. Furthermore, since $\left(1+\lambda^{2}\right)^{-\frac{1}{2}}=1+O\left(\lambda^{2}\right)$, the large $T$-behaviou of $V_{T}(N)$ is given by

$$
V_{T}(N)=1+\left(P_{T}\left(N+\frac{1}{T}\right)-P_{T}(N)\right)\left(2 P_{T}(N)-1\right)+O\left(T^{-2}\right)
$$

The unitary operator which describes the discrete time evolution of the wave operator,

$$
\Theta_{T}(N):=\Omega_{T}\left(N+\frac{1}{T}\right) \Omega_{T}^{*}(N)
$$

is easily shown to be

$$
\Theta_{T}(N)=U_{T}^{A^{*}}\left(N+\frac{1}{T}\right) V_{T}^{*}\left(N+\frac{1}{T}, N\right) U_{T}^{A}\left(N+\frac{1}{T}\right) .
$$

Instead of using $\Theta_{T}$ we shall make use of the operator $K_{T}(N)=T\left(1-\Theta_{T}(N)\right)$ and formulate equation (18) in the way $D \Omega_{T}=-\frac{1}{T} K_{T} \Omega_{T}$, or, summed up,

$$
\Omega_{T}\left(\frac{l}{T}\right)=1-\frac{1}{T} \sum_{n=0}^{l-1} K_{T}\left(\frac{n}{T}\right) \Omega_{T}\left(\frac{n}{T}\right)
$$

Note that $K_{T}(N)=O(1)$.

\subsection{The theorem about partial summing up}

The theorem and corollary of this section furnish the technical basis of this work.

Theorem 1 Let $W_{T}(N), N \in \frac{\mathrm{Z}}{T}$, be a sequence of unitaries satisfying the conditions of our set up, condition 1 with $k=1$, and suppose that $X(N)$ and $Y(N)$ are sequences of operators such that $D X(N)$ and $D Y(N)$ are of order $T^{-1}$. Let

$$
\tilde{X}(N)=-\frac{1}{2 \pi i} \int_{\Gamma_{T}(N)} R_{T}(N, z) X(N) R_{T}(N, z) d z
$$

where $R_{T}(N, z)$ is the resolvent of $W_{T}(N)$. Then

$$
\sum_{n=1}^{\ell} Q_{0} U_{T}^{A^{*}}\left(\frac{n}{T}\right) X\left(\frac{n}{T}\right) U_{T}^{A}\left(\frac{n}{T}\right) P_{0} Y\left(\frac{n}{T}\right)=\mathcal{B}+\frac{1}{T} \mathcal{S}
$$

where $P_{0}=P_{T}(0)$ and $Q_{0}=Q_{T}(0)$,

$$
\mathcal{B}=Q_{0} U_{T}^{A^{*}}(0) \tilde{X}\left(\frac{1}{T}\right) U_{T}^{A}\left(\frac{1}{T}\right) P_{0} Y\left(\frac{1}{T}\right)-Q_{0} U_{T}^{A^{*}}\left(\frac{l}{T}\right) \tilde{X}\left(\frac{l+1}{T}\right) U_{T}^{A}\left(\frac{l+1}{T}\right) P_{0} Y\left(\frac{l+1}{T}\right)
$$

is a boundary term and

$$
\mathcal{S}=\sum_{n=1}^{l} Q_{0} U_{T}^{A^{*}}\left(\frac{n}{T}\right)\left(Z\left(\frac{n}{T}\right) U_{T}^{A}\left(\frac{n}{T}\right) P_{0} Y\left(\frac{n}{T}\right)+\tilde{X}\left(\frac{n+1}{T}\right) W_{T}^{A}\left(\frac{n}{T}\right) U_{T}^{A}\left(\frac{n}{T}\right) P_{0} T D Y\left(\frac{n}{T}\right)\right)
$$

is a sum, and

$$
Z\left(\frac{n}{T}\right)=T\left(\left[\left(V_{T}^{*}\left(\frac{n}{T}\right)-1\right) W_{T}^{A}\left(\frac{n}{T}\right), \tilde{X}\left(\frac{n}{T}\right)\right]+D W_{T}^{A}\left(\frac{n-1}{T}\right) \tilde{X}\left(\frac{n}{T}\right)+D \tilde{X}\left(\frac{n}{T}\right) W_{T}^{A}\left(\frac{n}{T}\right)\right)
$$

Moreover, $Z\left(\frac{n}{T}\right)$ and $T D Y\left(\frac{n}{T}\right)$ remain bounded in the adiabatic limit. 
Proof: Note first the identity

$$
Q_{T}(N) X(N) P_{T}(N)=-Q_{T}(N)\left[W_{T}(N), \tilde{X}(N)\right] P_{T}(N)
$$

which follows from

$$
\begin{aligned}
{\left[W_{T}(N), \tilde{X}(N)\right] } & =-\frac{1}{2 \pi i} \int_{\Gamma_{T}(N)}\left[W_{T}(N)-z, R_{T}(N, z) X(N) R_{T}(N, z)\right] d z \\
& =\left[P_{T}(N), X(N)\right] .
\end{aligned}
$$

Substituting $W_{T}$ by the expression of the adiabatic time evolution one gets

$Q_{T}(N) X(N) P_{T}(N)=-Q_{T}(N)\left[W_{T}^{A}(N), \tilde{X}(N)\right] P_{T}(N)+Q_{T}(N)[A(N), \tilde{X}(N)] P_{T}(N)$

where $A=\left(V_{T}^{*}-1\right) W_{T}^{A}=O\left(T^{-1}\right)$. Using the intertwining properties (8) to obtain

$$
Q_{0} U_{T}^{A^{*}} X U_{T}^{A} P_{0}=Q_{0} U_{T}^{A^{*}}\left[W_{T}^{A}, \tilde{X}\right] U_{T}^{A} P_{0}+Q_{0} U_{T}^{A^{*}}[A, \tilde{X}] U_{T}^{A} P_{0}
$$

and the adiabatic equation of motion one arrives at

$$
\begin{aligned}
U_{T}^{A^{*}}\left(\frac{n}{T}\right)\left[W_{T}^{A}\left(\frac{n}{T}\right), \tilde{X}\left(\frac{n}{T}\right)\right] U_{T}^{A}\left(\frac{n}{T}\right) & =U_{T}^{A^{*}}\left(\frac{n}{T}\right) W_{T}^{A}\left(\frac{n-1}{T}\right) \tilde{X}\left(\frac{n}{T}\right) U_{T}^{A}\left(\frac{n}{T}\right)-U_{T}^{A^{*}}\left(\frac{n}{T}\right) \tilde{X}\left(\frac{n}{T}\right) U_{T}^{A}\left(\frac{n+1}{T}\right) \\
& +U_{T}^{A^{*}}\left(\frac{n}{T}\right)\left(W_{T}^{A}\left(\frac{n}{T}\right)-W_{T}^{A}\left(\frac{n-1}{T}\right)\right) \tilde{X}\left(\frac{n}{T}\right) U_{T}^{A}\left(\frac{n}{T}\right) \\
& =U_{T}^{A^{*}}\left(\frac{n-1}{T}\right) \tilde{X}\left(\frac{n}{T}\right) U_{T}^{A}\left(\frac{n}{T}\right)-U_{T}^{A^{*}}\left(\frac{n}{T}\right) \tilde{X}\left(\frac{n+1}{T}\right) U_{T}^{A}\left(\frac{n+1}{T}\right) \\
& +U_{T}^{A^{*}}\left(\frac{n}{T}\right) B\left(\frac{n}{T}\right) U_{T}^{A}\left(\frac{n}{T}\right)
\end{aligned}
$$

where

$$
B\left(\frac{n}{T}\right)=(D \tilde{X})\left(\frac{n}{T}\right) W_{T}^{A}\left(\frac{n}{T}\right)+\left(D W_{T}^{A}\right)\left(\frac{n-1}{T}\right) \tilde{X}\left(\frac{n}{T}\right) .
$$

By hypothesis are $D X$, and hence also $D \tilde{X}$, and $D W_{T}^{A}$ of order $T^{-1}$. Thus $Z\left(\frac{n}{T}\right)=T\left(\left[A\left(\frac{n}{T}\right), \tilde{X}\left(\frac{n}{T}\right)\right]+B\left(\frac{n}{T}\right)\right)$ is of order 1. Summing up yields the statement of the theorem. q.e.d.

Note that the sum $\mathcal{S}(24)$ is of the same form as the l.h.s. of (22). One might therefore apply the theorem iteratively if the boundary terms vanish. This is the content of the next corollary.

Corollary 1 Let in addition to the conditions of the last theorem $D^{(k)} W_{T}(N)$, $D^{(k)} X(N)$, and $D^{(k)} Y(N)$ be of order $T^{-k}$ for all $k$ and $X$ or $Y$ be supported inside $(0, s)$. Then for $l \geq s T$,

$$
\frac{1}{T} \sum_{n=1}^{l} Q_{0} U_{T}^{A^{*}}\left(\frac{n}{T}\right) X\left(\frac{n}{T}\right) U_{T}^{A}\left(\frac{n}{T}\right) P_{0} Y\left(\frac{n}{T}\right)=O\left(T^{-\infty}\right) .
$$


Proof: Note first that, if $D^{(k)} W_{T}, D^{(k)} X$, and $D^{(k)} Y$ are of order $T^{-k}$, then $D^{(k-1)} Z$ is of order $T^{-k+1}$. Therefore one can in this case apply the above theorem $k$ times iteratively each time gaining one order in $T^{-1}$, provided the boundary terms $\mathcal{B}$ in the r.h.s. of (22) vanish. We assert that this is the case under the assumptions on the support of $X$ or $Y$. For that, note that, for arbitrary $X$, $\operatorname{supp} X \subset[a, b]$, implies that $\operatorname{supp} D X \subset\left[a, b+\frac{1}{T}\right]$. By assumption, the support of $X$ or that of $Y$ is contained in $[\epsilon, s-\epsilon]$ for some $\epsilon>0$. This implies that $\operatorname{supp} Z \subset\left[\epsilon, s-\epsilon+\frac{1}{T}\right]$ or $\operatorname{supp} D Y \subset\left[\epsilon, s-\epsilon+\frac{1}{T}\right]$. Therefore, if $\epsilon>\frac{1}{T}$ then the boundary terms in the r.h.s. of (22) which could arise upon one-fold application of the theorem actually vanish. Inductively one concludes that if $T>\frac{k}{\epsilon}$ no boundary terms arise upon $k$-fold application of the theorem. Thus, after $k$-fold application of the theorem, the sum of interest has the form

$\frac{1}{T} \sum_{n=1}^{l} Q_{0} U_{T}^{A^{*}}\left(\frac{n}{T}\right) X\left(\frac{n}{T}\right) U_{T}^{A}\left(\frac{n}{T}\right) P_{0} Y\left(\frac{n}{T}\right)=\frac{1}{T^{k+1}} \sum_{i=1}^{2^{k}} \sum_{n=1}^{l} Q_{0} U_{T}^{A^{*}}\left(\frac{n}{T}\right) X_{i}\left(\frac{n}{T}\right) U_{T}^{A}\left(\frac{n}{T}\right) P_{0} Y_{i}\left(\frac{n}{T}\right)$,

where $X_{i}$ and $Y_{i}$ are sequences of bounded operators, provided $T>\frac{k}{\epsilon}$. In particular, the large $T$ behaviour of the sum is bounded by any power of $T^{-1}$. q.e.d.

\subsection{The first adiabatic theorem}

The first adiabatic theorem states that the physical time evolution is approximated by the adiabatic time evolution. It is obtained upon estimating the large $T$ behaviour of $\Omega_{T}$. For that we shall first investigate a sum of the form $\frac{1}{T} \sum_{n=0}^{l-1} K_{T}\left(\frac{n}{T}\right) Y\left(\frac{n}{T}\right)$ where $l$ is of the order of $T$.

Let us begin with the "diagonal" term $P_{0} K_{T}\left(\frac{n}{T}\right) P_{0} Y\left(\frac{n}{T}\right)$. Since, for any two projections $p, q$, holds $p(p-q) p=p(p-q)^{2} p$ equation (17) implies that $P_{T}(N)(1-$ $\left.V_{T}^{*}(N)\right) P_{T}(N)=O\left(T^{-2}\right)$, and therefore

$$
\begin{aligned}
\left\|\frac{1}{T} \sum_{n=0}^{[s T]} P_{0} K_{T}\left(\frac{n}{T}\right) P_{0} Y\left(\frac{n}{T}\right)\right\| & =\left\|\sum_{n=1}^{[s T]+1} U_{T}^{A *}\left(\frac{n}{T}\right) P_{T}\left(\frac{n}{T}\right)\left(1-V^{*}\left(\frac{n}{T}\right)\right) P_{T}\left(\frac{n}{T}\right) U_{T}^{A}\left(\frac{n}{T}\right) Y\left(\frac{n-1}{T}\right)\right\| \\
& \leq \frac{\text { const }}{T}\|Y\|_{s}
\end{aligned}
$$

where

$$
\|Y\|_{s}=\sup _{0 \leq n \leq s T}\left\|Y\left(\frac{n}{T}\right)\right\| .
$$

The same estimate holds for the norm of $\frac{1}{T} \sum_{n=0}^{[s T]} Q_{0} K_{T}\left(\frac{n}{T}\right) Q_{0} Y\left(\frac{n}{T}\right)$. To treat the "off diagonal" terms we apply Theorem 1 taking $X(N)=T\left(1-V_{T}^{*}(N)\right)=$ $T D P_{T}(N)\left(2 P_{T}(N)-1\right)+O\left(T^{-1}\right)$. In particular, $D X(N)$ is of order $T^{-1}$ in case $W_{T}$ satisfies condition 1 with $k \geq 2$. The terms of

$$
\frac{1}{T} \sum_{n=0}^{[s T]} Q_{0} K_{T}\left(\frac{n}{T}\right) P_{0} Y\left(\frac{n}{T}\right)=\frac{1}{T}\left(\mathcal{B}+\frac{1}{T} \mathcal{S}\right),
$$


where $\mathcal{B}$ and $\mathcal{S}$ are as in Theorem 1, are bounded from above by

$$
\|\mathcal{B}\| \leq \text { const }\|Y\|_{s}
$$

and

$$
\|\mathcal{S}\| \leq \text { const } T\|\mid Y\|_{s}
$$

with

$$
\|Y\|_{s}=\max \left\{\|Y\|_{s}, T\|D Y\|_{s}\right\}
$$

and $T$-independent constants. Again, the same estimate holds for the norm of the other "off diagonal" $\frac{1}{T} \sum_{n=0}^{[s T]} P_{0} K_{T}\left(\frac{n}{T}\right) Q_{0} Y\left(\frac{n}{T}\right)$. Summarizing we obtain

$$
\left\|\frac{1}{T} \sum_{n=0}^{[s T]} K_{T}\left(\frac{n}{T}\right) Y\left(\frac{n}{T}\right)\right\| \leq \frac{\text { const }}{T}\|\mid Y\| \|_{s} .
$$

Theorem 2 If $W_{T}(N), N \in \frac{\mathbf{Z}}{T}$, is a sequence of unitaries satisfying the conditions of our set up, condition 1 with $k \geq 2$, then

$$
\Omega_{T}(N)=1+O\left(T^{-1}\right)
$$

Proof: Define $\Omega_{0}:=1$ and, for $j \geq 1$,

$$
\Omega_{j}\left(\frac{l}{T}\right):=-\frac{1}{T} \sum_{n=0}^{l-1} K_{T}\left(\frac{n}{T}\right) \Omega_{j-1}\left(\frac{n}{T}\right) .
$$

Then, by (32) we have, for $j \geq 1$,

$$
\left\|\Omega_{j}\left(\frac{l}{T}\right)\right\| \leq \frac{\text { const }}{T}\left\|\mid \Omega_{j-1}\right\|_{\frac{l}{T}} .
$$

But $D \Omega_{j}=-\frac{1}{T} K_{T} \Omega_{j-1}$ so that, for $j \geq 1$,

$$
\left\|\mid \Omega_{j}\right\|_{s}=\max \left\{\left\|\Omega_{j}\right\|_{s},\left\|\Omega_{j-1}\right\|_{s}\right\} .
$$

Since $\left\|\Omega_{0}\right\|_{s}=1$ and hence, by (35), $\left\|\Omega_{1}\right\|_{s}=O\left(T^{-1}\right)$ one obtains by induction on $j$, for all $j>1$,

$$
\left\|\Omega_{j}\right\|_{s}=O\left(T^{1-j}\right) .
$$

Furthermore, let

$$
R_{M}:=\Omega_{T}-\sum_{j=0}^{M} \Omega_{j}
$$

Then

$$
\begin{aligned}
R_{M}\left(\frac{l}{T}\right) & =-\frac{1}{T} \sum_{n=0}^{l-1} K_{T}\left(\frac{n}{T}\right) \Omega_{T}\left(\frac{n}{T}\right)+\frac{1}{T} \sum_{j=1}^{M} \sum_{n=0}^{l-1} K_{T}\left(\frac{n}{T}\right) \Omega_{j-1}\left(\frac{n}{T}\right) \\
& =-\frac{1}{T} \sum_{n=0}^{l-1} K_{T}\left(\frac{n}{T}\right) R_{M-1}\left(\frac{n}{T}\right)
\end{aligned}
$$


which shows that $R_{M}$ satisfies the same equation as $\Omega_{k}$, hence, for $M \geq 1$,

$$
\left\|R_{M}\left(\frac{l}{T}\right)\right\| \leq \frac{\text { const }}{T}\left\|\mid R_{M-1}\right\|_{\frac{l}{T}} .
$$

Since $R_{0}=\Omega_{T}-1$ is of order 1 in $T$ and $D R_{0}=-\frac{1}{T} K_{T} \Omega_{T}$ is of order $T^{-1}$ the above formula shows that $R_{1}$ is of order $T^{-1}$ and, analogously, for all $M>1$,

$$
\left\|R_{M}\right\|_{s}=O\left(T^{1-M}\right) \text {. }
$$

As $\Omega_{T}=1+R_{1}+\Omega_{1}$, and $\left\|\Omega_{T}(N)-1\right\| \leq\left\|\Omega_{T}-1\right\|_{N}$, the statement of the theorem follows.

q.e.d.

\subsection{The second adiabatic theorem}

We have just seen that the adiabatic time evolution approximates the physical time evolution. By construction, it preserves the spectral subspace corresponding to the projection $P_{T}(N)$. The quantities $\left\|Q_{0} \Omega_{T} P_{0}\right\|$ and $\left\|P_{0} \Omega_{T} Q_{0}\right\|$ are therefore upper bounds to the probabilities for the physical system to tunnel out and into, respectively, that spectral subspace. In case $D^{(k)} W_{T}(N)$ is of order $T^{-k}$ for all $k$ we shall show now that these probabilities are smaller than any inverse power of the time scale. For the remainder of this section we assume that $W_{T}(N), N \in \frac{\mathrm{Z}}{T}$, is a sequence of unitaries satisfying the conditions of our set up, but condition 1 for all $k \geq 1$.

Recall that $\Omega_{0}=1$ and, for $j \geq 1$,

$$
Q_{0} \Omega_{j}\left(\frac{l}{T}\right) P_{0}=-\frac{1}{T} \sum_{n=1}^{l} Q_{0} K_{T}\left(\frac{n-1}{T}\right) \Omega_{j-1}\left(\frac{n-1}{T}\right) P_{0} .
$$

Lemma $1 F i x$ an integer $j \geq 1$. Let $X$ be supported inside $(0, s)$ and $D^{k} X(N)=$ $O\left(T^{-k}\right)$ for all $k$, and assume that, for $l \geq s T$,

$$
Q_{0} \Omega_{m}\left(\frac{t}{T}\right) P_{0}=O\left(T^{-\infty}\right) \text { for all } 0 \leq m \leq j-1 .
$$

Then, for all $1 \leq j^{\prime} \leq j$ holds

$$
\begin{aligned}
& \sum_{n=1}^{l} Q_{0} U_{T}^{A^{*}}\left(\frac{n}{T}\right) X\left(\frac{n}{T}\right) U_{T}^{A}\left(\frac{n}{T}\right) \Omega_{j^{\prime}-1}\left(\frac{n}{T}\right) P_{0} \\
& =\gamma\left(j^{\prime}\right) \frac{1}{T} \sum_{n=1}^{l} Q_{0} U_{T}^{A^{*}}\left(\frac{n}{T}\right) Z\left(\frac{n}{T}\right) U_{T}^{A}\left(\frac{n}{T}\right) \Omega_{j^{\prime}-1}\left(\frac{n}{T}\right) P_{0} \\
& +\gamma\left(j^{\prime}-1\right) \frac{1}{T} \sum_{n=1}^{l} Q_{0} U_{T}^{A^{*}}\left(\frac{n}{T}\right) Z_{1}\left(\frac{n}{T}\right) U_{T}^{A}\left(\frac{n}{T}\right) \Omega_{j^{\prime}-2}\left(\frac{n}{T}\right) P_{0}+O\left(T^{-\infty}\right),
\end{aligned}
$$

where $\gamma\left(j^{\prime}\right)=1$ for $j^{\prime}>1$ and $\gamma\left(j^{\prime}\right)=0$ otherwise,

$$
Z_{1}\left(\frac{n}{T}\right)=\tilde{X}\left(\frac{n+1}{T}\right) W_{T}^{A}\left(\frac{n}{T}\right) P_{T}\left(\frac{n}{T}\right) T\left(V_{T}^{*}\left(\frac{n-1}{T}\right)-1\right),
$$


and $Z$ and $\tilde{X}$ are the same as in Theorem 1 , cf. (21), (25). Furthermore, $D^{k} Z\left(\frac{n}{T}\right)=O\left(T^{-k}\right)$ and $D^{k} Z_{1}\left(\frac{n}{T}\right)=O\left(T^{-k}\right)$ for all $k$.

Proof: For $j^{\prime}=1$ the announced result is a special case of Corollary 1, namely that where $Y(N)=1$ for all $N$. So let us consider the case $j^{\prime}>1$. Since $Q_{0} \Omega_{j^{\prime}-1}\left(\frac{n}{T}\right) P_{0}=O\left(T^{-\infty}\right)$,

$$
\begin{aligned}
& \sum_{n=1}^{l} Q_{0} U_{T}^{A^{*}}\left(\frac{n}{T}\right) X\left(\frac{n}{T}\right) U_{T}^{A}\left(\frac{n}{T}\right) \Omega_{j^{\prime}-1}\left(\frac{n}{T}\right) P_{0} \\
& =\sum_{n=1}^{l} Q_{0} U_{T}^{A^{*}}\left(\frac{n}{T}\right) X\left(\frac{n}{T}\right) U_{T}^{A}\left(\frac{n}{T}\right) P_{0} \Omega_{j^{\prime}-1}\left(\frac{n}{T}\right) P_{0}+O\left(T^{-\infty}\right) \\
& =\frac{1}{T} \sum_{n=1}^{l} Q_{0} U_{T}^{A^{*}}\left(\frac{n}{T}\right) Z\left(\frac{n}{T}\right) U_{T}^{A}\left(\frac{n}{T}\right) \Omega_{j^{\prime}-1}\left(\frac{n}{T}\right) P_{0} \\
& +\frac{1}{T} \sum_{n=1}^{l} Q_{0} U_{T}^{A^{*}}\left(\frac{n}{T}\right) \tilde{X}\left(\frac{n+1}{T}\right) W_{T}^{A}\left(\frac{n}{T}\right) U_{T}^{A}\left(\frac{n}{T}\right) P_{0} T D \Omega_{j^{\prime}-1}\left(\frac{n}{T}\right) P_{0}+O\left(T^{-\infty}\right)
\end{aligned}
$$

the last equality following by Theorem 1 with $Y\left(\frac{n}{T}\right)=\Omega_{j^{\prime}-1}\left(\frac{n}{T}\right) P_{0}$ provided $T$ is large enough. In fact, as for Corollary 1 one shows that the boundary terms vanish if $T>\frac{1}{\epsilon}$ where $\epsilon>0$ is chosen such that $\operatorname{supp} X(N) \subset[\epsilon, s-\epsilon]$. Using that, for $j^{\prime}>1, D \Omega_{j^{\prime}-1}\left(\frac{n}{T}\right) P_{0}=-U_{T}^{A^{*}}\left(\frac{n}{T}\right)\left(1-V_{T}^{*}\left(\frac{n-1}{T}\right)\right) U_{T}^{A}\left(\frac{n}{T}\right) \Omega_{j^{\prime}-2}\left(\frac{n}{T}\right) P_{0}$ and the intertwining properties (8) one gets

$$
\begin{aligned}
U_{T}^{A}\left(\frac{n}{T}\right) P_{0} T D \Omega_{j^{\prime}-1}\left(\frac{n}{T}\right) P_{0} & =U_{T}^{A}\left(\frac{n}{T}\right) P_{0} U_{T}^{A^{*}}\left(\frac{n}{T}\right) T\left(V_{T}^{*}\left(\frac{n-1}{T}\right)-1\right) U_{T}^{A}\left(\frac{n}{T}\right) \Omega_{j^{\prime}-2}\left(\frac{n}{T}\right) P_{0} \\
& =P_{T}\left(\frac{n}{T}\right) T\left(V_{T}^{*}\left(\frac{n-1}{T}\right)-1\right) U_{T}^{A}\left(\frac{n}{T}\right) \Omega_{j^{\prime}-2}\left(\frac{n}{T}\right) P_{0} .
\end{aligned}
$$

This shows (41) for $j^{\prime}>2$. If $j^{\prime}=2$ then we may apply Corollary 1 to the term containing $\Omega_{j^{\prime}-2}\left(\frac{n}{T}\right)$ obtaining that this term is $O\left(T^{-\infty}\right)$. It was already shown in the proof of Corollary 1 that $D^{k} Z\left(\frac{n}{T}\right)=O\left(T^{-k}\right)$. That $D^{k} Z_{1}\left(\frac{n}{T}\right)=O\left(T^{-k}\right)$ follows from the discrete Leibniz rule, since all factors satisfy this condition.q.e.d.

Theorem 3 Suppose that $W_{T}(N)$ satisfies the conditions 1 and 2 for all $k$ and that $V_{T}^{*}(N)-1$ be supported within $(0, s)$. Then for $N \notin(0, s)$

$$
Q_{0} \Omega_{T}(N) P_{0}=O\left(T^{-\infty}\right) .
$$

Proof: Recall that, for all $M, Q_{0} \Omega_{T} P_{0}=Q_{0} R_{M} P_{0}+\sum_{j=1}^{M} Q_{0} \Omega_{j} P_{0}$. We show by induction on $j$ that, for $l \geq s T$ and $j \geq 1$,

$$
Q_{0} \Omega_{j}\left(\frac{l}{T}\right) P_{0}=O\left(T^{-\infty}\right) .
$$

The theorem then follows from (38). (44) is true for $j=1$, since, by Corollary 1 applied to $X=1-V_{T}^{*}$ and $Y=1$,

$$
\begin{aligned}
Q_{0} \Omega_{1}\left(\frac{l}{T}\right) P_{0} & =-\frac{1}{T} \sum_{n=1}^{l} Q_{0} K_{T}\left(\frac{n-1}{T}\right) P_{0} \\
& =-\sum_{n=1}^{l} Q_{0} U_{T}^{A^{*}}\left(\frac{n}{T}\right)\left[1-V_{T}^{*}\left(\frac{n}{T}\right)\right] U_{T}^{A}\left(\frac{n}{T}\right) P_{0}=O\left(T^{-\infty}\right) .
\end{aligned}
$$


To prove (44) for $j>1$ we assume (40) and use Lemma 1 repeatedly. In view of (39) we apply Lemma 1 first with $X=V_{T}^{*}-1$, and in each further step with $X$ being separately equal to the arising $Z$ 's and $Z_{1}$ 's from the last step. This is possible as long as the resulting $Z$ 's and $Z_{1}$ 's satisfy also the remaining condition for the lemma, that is, as long as they have their support inside $(0, s)$. But as for Corollary 1 one shows that this is indeed the case if $T>\frac{k}{\epsilon}$, where $\operatorname{supp} X(N) \subset[\epsilon, s-\epsilon]$ for all $N$. Thus if $T>\frac{k}{\epsilon}, k$-fold application of Lemma 1 results in

$$
Q_{0} \Omega_{j}\left(\frac{l}{T}\right) P_{0}=\frac{1}{T^{k}} \sum_{i=1}^{\min \{k, j-1\}} \sum_{n=1}^{l} Q_{0} U_{T}^{A^{*}}\left(\frac{n}{T}\right) X_{i}\left(\frac{n}{T}\right) U_{T}^{A}\left(\frac{n}{T}\right) \Omega_{j-i}\left(\frac{n}{T}\right) P_{0}+O\left(T^{-\infty}\right),
$$

where each $X_{i}$ contains at most $2^{k}$ terms which all remain bounded in the adiabatic limit. Since also all $\Omega_{j}$ remain bounded in the adiabatic limit it follows that $Q_{0} \Omega_{j}\left(\frac{l}{T}\right) P_{0}=O\left(T^{-k}\right)$ for all $k$.

q.e.d.

That $P_{0} \Omega_{T}(N) Q_{0}=O\left(T^{-\infty}\right)$ is proven likewise.

Acknowledgement. The authors wish to thank Markus Klein for helpful discussions. This work was supported by the Deutsche Forschungsgemeinschaft.

\section{References}

[1] J. Avron, R. Seiler and L. Yaffe: Adiabatic theorems and applications to the quantum Hall effect. Commun. Math. Phys. 110, 33-49 (1987)

[2] M.V. Berry: Quantal phase factors accompanying adiabatic changes. Proc. R. Soc. Lond. A392, 45-57 (1984)

[3] A. Bobenko and U. Pinkall: Discrete Surfaces with Constant Negative Gaussian Curvature and the Hirota Equation. SFB 288 preprint No. 127, to appear in J. Diff. Geom.

[4] M. Born and V. Fock: Beweis des Adiabatensatzes. Z. Phys. 51, 165-169 (1928)

[5] T. Kato: On the adiabatic theorem of quantum mechanics. J. Phys. Soc. J. Jpn. 5, 435-439 (1950)

[6] T. Kato: Perturbation theory for linear operators. Springer-Verlag, Berlin/Heidelberg, 1966

[7] M. Klein and R. Seiler: Power-low corrections to the Kubo formula vanish in quantum Hall system. Commun. Math. Phys. 128, 141-160 (1990)

[8] B. Simon: Holonomy, the quantum adiabatic theorem and Berry's phase. Phys. Rev. Lett. 51, 2167-2170 (1983) 


\section{Preprints are availlable at: http://www-sfb288.math.tu-berlin.de List of most recent 50 sfb288 preprints}

210 M. Lüdde: A Cellular Braid Action and the Yang-Baxter Equation

211 K. Große-Braukmann, K. Polthier: Constant Mean Curvature Surfaces Derived from Delaunay`s and Wente`s Examples

212 H. Baum, I. Kath: Normally hyperbolic operators, the Huygens property and conformal geometry

213 V. Bach, J. Fröhlich, I. Sigal: Quantum Electrodynamics of Confined Non-Relativistic Particles

214 H. Baum: The Dirac operator on Lorentzian spin manifolds and the Huygens property

215 A.G. Bytsko: Fusion of q-tensor operators: quasi-Hopf-algebraic point of view

216 M. Karowski, R. Schrader, E. Vogt: Invariants of 3-manifolds, unitary representations of the mapping class group and numerical calculations

217 P. Nurowski: Twistor Bundles, Einstein Equations and real Structures

218 U. Eitner: Schlesinger Transformations for Bonnet Surfaces

219 A. Schmitt: The Energy Function of the Skyrme Model

220 W. Müller, M. Schmidt, R. Schrader: Hyperelliptic Riemann surfaces of infinite genus and solutions of the $\mathrm{KdV}$ equation

221 J. Brüning: The Signature Theorem for Manifolds with Metric Horns

222 J. Brüning, M. Lesch: On the eta-invariant of certain non-local boundary value problems

223 J. Dorfmeister, G. Haak: On constant mean curvature surfaces with periodic metric

224 C. Figurei de Morisson Faria, A. Fring, R. Schrader: On the Influence of Pulse Shapes on Ionization Probability

225 B.D. Dörfel, S. Meißner: Finite-size corrections of an integrable chain with alternating spins

226 G. Haak: On a theorem by do Carmo and Dajczer

227 B. Schroer: Notes on the Wigner Representation Theory of the Pioncaré Group, Localization and Statistics

228 B. Schroer: Motivations and Physical Aims of Algebraic QFT

229 J. Kellendonk: Topological Equivalence of Tilings

230 Thomas Schmitt: The Cauchy Problem for Abstract Evolution Equations with Ghost and Fermion Degrees of Freedom

231 N. Kutz: Free massive fermions inside the quantum discrete sine-Gordon model

232 A. Knauf, Y.G. Sinai: Classical Nonintegrability, Quantum Chaos

233 M. Pflaum, M. Schottenloher: Holomorphic deformation of Hopf algebras and applications to quantum groups

234 F. Nill: On the Structure of Momodromy Algebras and Drinfeld Doubles

235 H. Babujian, M. Karowski, A. Zapletal: SU(N) Matrix Difference Equations and a Nested Bethe Ansatz 
236 H. Babujian, M. Karowski, A. Zapletal: U(N) Matrix Differende Equations and a Nested Bethe Ansatz

237 J. Weber: Relation between Morse Theory of closed Geodesics of S1 and Symplectic Floer Theory of $\mathrm{T}^{*} \mathrm{~S} 1$

238 A. Bytsko, V. Schomerus: Vertex Operators - From a Toy Model to Lattice Algebras

239 U. Hertrich-Jeromin, T. Hoffmann, U. Pinkall: A discrete version of the Darboux transform for isothermic surfaces

240 A. Moroianu: On the Infinitesimal Isometries of Manifolds with Killing Spinors

241 A. Moroianu: Parallel and Killing Spinors on SpinC Manifolds

242 A. Belavin, A. Fring: On the Fermionic Quasi-particle Interpretation in Minimal Models of Conformal Field

243 A. Moroianu: Complex Contact Structures and Spin^c Manifolds

$244 \mathrm{H}$. Baumgaertel, F. Lledo: Superselection structures for $\mathrm{C}^{*}$-algebras with nontrivial center

245 M.U. Schmidt, A.P. Veselov: Elliptic Calogero Moser quantum problem and deformations of algebraic surfaces

246 C. Binnenhei: O Realized on Bose Fock Space

247 K. Mohnke: On Fillings by Holomorphic Discs of the Levels of a Morse Function

248 E. Akkermans, J.E. Avron, R. Narevich, R. Seiler: Boundary Conditions for Bulk and Edge States in Quantum Hall Systems

249 J.C.A. Barata, F. Nill: Dyonic Sectors and Intertwiner Connections in 2+1-dimensional Lattice ZN-Higgs Models

$250 \mathrm{H}$. Baum: Strictly pseudoconvex spin manifolds, Fefferman spaces and Lorentzian twistor spinors

251 T. Friedrich, A. Trautman: Clifford structures and spinor bundles

252 Piotr G. Grinevich, Martin U. Schmidt: Conformal invariant functionals of immersions of tori R3

253 Ines Kath: Pseudo-Riemannian T-duals of compact Riemannian reductive spaces

254 P. G. Grinevich, M. U. Schmidt: Closed curves in R3: a characterization in terms of curvature and torsion, the Hasimoto map and periodic solutions of the Filament Equation

255 D. Guido, R. Longo, H.-W. Wiesbrock: Extensions of Conformal Nets and Superselection Structures

256 F. Duzaar, E. Kuwert: Minimization of Conformally Invariant Energies in Homotopy Classes

257 F. Duzaar, A. Gastel: Minimizing p-Harmonic Maps at a Free Boundary

258 Y. M. Berezansky: Commutative Jacobi Fields in Fock Space

259 A. Dranov, J. Kellendonk, R. Seiler: Discrete time adiabatic theorems for quantum mechanical systems 